\title{
EMPODERAMIENTO MEDIÁTICO MEDIANTE E-LEARNING. DISEÑO Y VALIDACIÓN DE UNA ESCALA
}

\section{Media empowerment through e-learning. Design and validation of a scale}

Juan-Francisco Martínez-Cerdá y Joan Torrent-Sellens

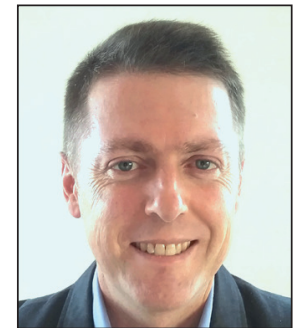

Juan-Francisco Martínez-Cerdá, PhD candidate, investiga el desarrollo de habilidades orientadas a la empleabilidad de los trabajadores, a través de sistemas sociotécnicos (STS) relacionados con e-learning. Fue investigador en la Universitat Autònoma de Barcelona (UAB) y jefe de estudios y proyectos en diversas empresas del ámbito público y privado. Mantiene vinculación con el Grupo Interdisciplinario de Investigación sobre las TIC (i2TIC) desde 2009.

http://orcid.org/0000-0002-6711-4956

jmartinezcer@uoc.edu

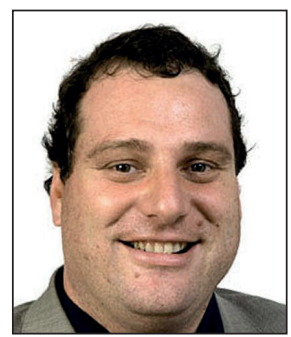

Joan Torrent-Sellens es director del Grupo Interdisciplinario de Investigación sobre las TIC (i2TIC) y profesor agregado de Estudios de Economía y Empresa de la Universitat Oberta de Catalunya (UOC). Sus líneas de investigación son la economía del conocimiento, el emprendimiento y la innovación, la empresa y el trabajo en red, las fuentes de productividad, competitividad y crecimiento económico, y la salud y el bienestar, materias sobre las que ha publicado numerosos artículos en revistas nacionales e internacionales, así como diversos libros.

http://orcid.org/0000-0002-6071-422X

jtorrent@uoc.edu

Universitat Oberta de Catalunya (UOC) Av. Tibidabo, 39-43. 08035 Barcelona, España

\section{Resumen}

Las actuales facilidades para la educación por medios online permiten adquirir las competencias profesionales y personales demandadas para el siglo XXI. En este artículo se estudia el empoderamiento mediático de los alumnos como ciudadanos que usan múltiples medios de comunicación. Se establece la primera escala para la medida de dicho empoderamiento, analizando el ejercicio de la ciudadanía activa y el uso de diversos medios de comunicación, como habilidades adquiridas mediante e-learning. El estudio se realizó con antiguos estudiantes de la Universitat Oberta de Catalunya ( $\mathrm{n}=544)$, construyéndose una escala consistente y fiable para las dos subescalas que la conforman: ciudadanía activa $(5$ items, $\alpha=0,93)$ y uso diversificado de los medios ( 3 items, $\alpha=0,95$ ).

\section{Palabras clave}

E-learning; Educación online; Alfabetización mediática; Ciudadanía activa; Empoderamiento digital; Empoderamiento mediático; Inclusión social; Pensamiento crítico; Medios de comunicación; Sociedad civil; Escala; Encuesta.

\begin{abstract}
The current ease of online education allows students to acquire the professional and personal competencies demanded for the XXI century. In this article we study the media empowerment of students, as citizens, using multiple means of communication. We have established the first scale for the measurement of such empowerment, analyzing the exercise of active citizenship and the use of various media, as skills acquired through e-learning. The study was carried out with alumni of the Universitat Oberta de Catalunya $(n=544)$, constructing a consistent and reliable scale for the two subscales that comprise it: active citizenship ( 5 items, $\alpha=0.93$ ) and diversified use of media ( 3 items, $\alpha=0.95$ ).
\end{abstract}

\section{Keywords}

E-learning; Online education; Media literacy; Active citizenship; Digital empowerment; Media empowerment; Social inclusion; Critical thinking; Media; Civil society; Scale; Survey. 
Martínez-Cerdá, Juan-Francisco; Torrent-Sellens, Joan (2017). “Empoderamiento mediático mediante e-learning. Diseño y validación de una escala". El profesional de la información, v. 26, n. 1, pp. 43-54.

https://doi.org/10.3145/epi.2017.ene.05

\section{Introducción}

La situación socioeconómica global desde la primera década del siglo XXI ha provocado que diferentes sectores de la población hayan perdido tanto su empleo como parte de sus derechos ciudadanos. En Europa se ha incrementado el riesgo de exclusión social por factores como la crisis financiera o las migraciones provocadas por situaciones de guerra de países vecinos. Los últimos datos oficiales (de 2014) apuntan a que más de 122 millones de personas están en riesgo de pobreza y exclusión social (Eurostat, 2016). Han contribuido a ello factores socioculturales, así como la pérdida de habilidades y competencias para la participación en la vida social y laboral, en la que han ocurrido vertiginosos cambios tecnológicos (Lenger; Schumacher, 2015).

La educación ha emergido ante esta situación como una solución en diversas acciones políticas y legislativas en Europa, como Key competences for lifelong learning y Enhancing digital literacy, skills and inclusion (European Union, 2016). Bajo este punto de vista, la educación online, de la mano de las competencias mediáticas y de su misión orientada hacia el cambio social, puede contribuir a la adquisición de habilidades válidas para la empleabilidad, la integración social, y la participación activa en la economía del conocimiento (Park, 2012; Tait, 2013).

Desde el sector del e-learning se realizan contribuciones para incrementar las competencias informacionales de los estudiantes, como las ejecutadas por la Biblioteca de la Universidad Nacional de Educación a Distancia (UNED) (Moreno-Pulido; Sánchez-Fernández, 2015). Desde la vertiente online, y mediante acciones de alfabetización mediática dirigidas a la inserción laboral y social, estas iniciativas profundizan en la labor social integradora de las bibliotecas públicas (Sánchez-García; Yubero, 2015). También se detectan iniciativas de e-learning para el empoderamiento del sector público (Hur; Im, 2013).

Estas actuaciones de la educación online pueden ayudar a frenar hechos tan preocupantes como que los medios de comunicación sean paulatinamente propiedad de menos organizaciones y empresas, o de que esté produciéndose una unificación de fuentes de información. Estas acciones, que podrían anular el razonamiento propio e individual de las personas (Martínez-Pérez, 2016), son ingredientes fundamentales del empoderamiento y el pleno ejercicio de los derechos de los ciudadanos.

La presente investigación estudia la adquisición mediante e-learning de habilidades de la ciudadanía activa y el uso diversificado de los medios de comunicación, presentando la primera escala validada de este tipo. El artículo considera los modelos emergentes de educación online, como los moocs, basados en medios sociales y configurados para ser instrumentos educomunicativos que contribuyan al cambio social necesario para la mejora de la sociedad (Gil-Quintana, 2016). Esta apuesta se aproxima a las ideas de empoderamiento, emancipación, transformación, y acción social para la escucha de los oprimidos planteadas por Freire (1970).

El texto es afín a Martens (2013), quien señalaba que hacían falta más estudios empíricos sobre alfabetización mediática, tras haber examinado más de 150 investigaciones. Cabe recordar el histórico nexo existente entre este campo del conocimiento y el empoderamiento mediático (Unesco, 1982).

\section{La educación online, mediante nuevos modelos pedagógicos basados en me- dios sociales, permite adquirir compe- tencias que ayudan al empoderamiento ciudadano}

\section{Marco analítico}

\subsection{Educación online y empoderamiento mediático}

A nivel teórico esta investigación enlaza con el aprendizaje de competencias llevado a cabo a lo largo de la vida y planteado por la teoría del capital humano (Schultz, 1961; Becker, 1993). Su orientación hacia el aprendizaje online es necesaria tras observar los actuales y futuros entornos para la adquisición de habilidades (profesionales, sociales, personales, etc.), con pedagogías basadas en internet que son globales, abiertas, adaptables, centradas en el estudiante, interactivas, sociales e ininterrumpidas (Varis; Puukko, 2010; Redecker et al., 2011).

El empoderamiento, originado en EUA ante la "ineficacia de la política -implementada en los años setenta- que fortalecía instituciones y olvidaba a las personas" (Montero, 2003, p. 71), es afín e intrínseco al contexto educativo, mediante el constructivismo social. Esta teoría se basa en la adición personal de nuevos conocimientos y experiencias sobre la base de los anteriores (Piaget, 2000), todo ello dentro del contexto de interacciones sociales entre estudiantes y profesores (Vygotsky, 1997).

Según esta perspectiva, esta dialéctica es la base del empoderamiento de los estudiantes, que puede ser realizado tanto mediante educomunicación (Hobbs, 2011) como mediante e-learning (Onah, 2012). La participación ciudadana y el empoderamiento digital son vitales para lograr la inclusión social de los ciudadanos (Mäkinen, 2006). En este campo se detectan lazos entre la educomunicación, la ciudadanía y la construcción de su autonomía mediática (Rivoltella, 2005; Gozálvez-Pérez; Aguaded-Gómez, 2012). Dias-Fonseca y Potter (2016) inciden en la importancia de la ciudadanía online y su vinculación a la alfabetización mediática. 
En el área común de la educomunicación, el empoderamiento, y la ciudadanía democrática, emergen propuestas teóricas que plantean y proporcionan dimensiones e indicadores tanto similares como complementarios (Celot; Pérez-Tornero, 2009; Ferrés-Prats; Piscitelli, 2012; Pérez-Rodríguez; Delgado-Ponce, 2012; Román-García; Almansa-Martínez; Cruz-Díaz, 2016). No obstante, no se están enfocadas a la validación psicométrica de escalas.

\subsection{Escalas psicométricas de alfabetización mediáti- ca y educación online}

Pese a la existencia de numerosas escalas sobre alfabetización mediática, empoderamiento y ciudadanía, no se ha llevado a cabo una escala que relacione dichos campos con el e-learning. Estas mediciones se orientan hacia la alfabetización mediática a nivel general, su perspectiva educativa, y su influencia sobre aspectos relacionados con la salud.

Respecto a dimensiones y factores relacionados explícitamente con el empoderamiento y la ciudadanía democrática, Duran et al. (2008) tienen en cuenta la participación mediática activa; Akkoyunlu, Yilmaz-Soylu y Caglar (2010) diseñan una escala para el empoderamiento digital de los estudiantes universitarios, y Choi (2016) y Zhang y Zhu (2016) tienen en cuenta la ciudadanía participativa.

En escalas de educación online, Van-de-Vord (2010) estudia la alfabetización informacional, y Prior et al. (2016) exploran la actitud, alfabetización digital y eficacia del comportamiento de aprendizaje online. Otras aproximaciones posteriores trabajan sobre estos conceptos, pero sin proponer ninguna escala (Ng, 2012; Pineda-Báez et al., 2012; Tang; Tseng, 2013; Kratochvil, 2014; Tury; Robinson; Bawden, 2015).

Tabla 1. Preguntas y respuestas del cuestionario

\begin{tabular}{|c|c|c|}
\hline \multicolumn{3}{|c|}{$\begin{array}{l}\text { Respecto a los medios de comunicación y las noticias, la educación online finalizada me ha ayudado a... } \\
\text { Piense que se trata de valorar si la educación online ha tenido un impacto importante, o no. }\end{array}$} \\
\hline Dimensión propuesta & Items & Dimensiones y autores \\
\hline Ciudadanía activa & $\begin{array}{l}\text { - Participar más activamente en la } \\
\text { vida civil y política. } \\
\text { _ } \quad \text { Actuar socialmente frente a aspectos } \\
\text { de la vida que veo que no funcionan } \\
\text { correctamente. } \\
\text { - } \quad \text { Sentirme más integrado en grupos, } \\
\text { comunidades, asociaciones, etc. } \\
\text { - Ganar más conocimiento sobre mis } \\
\text { derechos como ciudadano. } \\
\text { - Crear mensajes y contenidos pro- } \\
\text { pios, relacionados con las noticias. }\end{array}$ & $\begin{array}{l}\text { Estructuras y participación (Duran et al., 2008) } \\
\text { Participación ciudadana (Celot; Pérez-Tornero, 2009) } \\
\text { Comunicación y ética (Chang et al., 2011) } \\
\text { Acción (Levin-Zamir; Lemish; Gofin, 2011) } \\
\text { Interacción, producción y difusión (Ferrés-Prats; Piscitelli, 2012) } \\
\text { Participación ciudadana, y comunicación (Pérez-Rodríguez; Delgado-Ponce, } \\
\text { 2012) } \\
\text { Producción (Ashley; Maksl; Craft, 2013) } \\
\text { Uso (Koc; Barut, 2016) } \\
\text { Lenguaje, recepción e interacción, ideología y valores (Román-García; } \\
\text { Almansa-Martínez; Cruz-Díaz, 2016) } \\
\text { Alfabetización mediática (Vraga et al., 2016) } \\
\text { Ciudadanía participativa (Zhang; Zhu, 2016) }\end{array}$ \\
\hline $\begin{array}{l}\text { Uso diversificado de } \\
\text { medios de comuni- } \\
\text { cación }\end{array}$ & $\begin{array}{l}\text { - Usar más medios de comunicación. } \\
\text { - Conocer más medios de comunica- } \\
\text { - Utión. } \\
\text { - Utizar eficazmente los medios de } \\
\text { comunicación. }\end{array}$ & $\begin{array}{l}\text { Recursos (Norman; Skinner, 2006) } \\
\text { Entendimiento, acceso y comprensión (Arke; Primack, 2009) } \\
\text { Uso equilibrado y activo de los medios, y conocimiento sobre los medios y su } \\
\text { regulación (Celot; Pérez-Tornero, 2009) } \\
\text { Autores y audiencias, medios y significados, realidad y representación (Bier et } \\
\text { al., 2011) } \\
\text { Aprendizaje (Chang et al., 2011) } \\
\text { Identificación (Levin-Zamir; Lemish; Gofin, 2011) } \\
\text { Búsqueda, procesado y eficacia (Claro et al., 2012) } \\
\text { Tecnología, interacción, producción y difusión, ideología y valores, y estética } \\
\text { (Ferrés-Prats; Piscitelli, 2012) } \\
\text { Acceso y búsqueda, tecnología, ideología y valores (Pérez-Rodríguez; } \\
\text { Delgado-Ponce, 2012) } \\
\text { Producción y consumo (Ashley; Maksl; Craft, 2013) } \\
\text { Conocimiento e industria, control, estructuras y uso (Potter, 2010; Maksl; } \\
\text { Ashley; Craft, 2015) } \\
\text { Uso (Koc; Barut, 2016) } \\
\text { Autores y audiencias, mensajes y significados, representación y realidad } \\
\text { (Phelps-Tschang et al., 2015) } \\
\text { Lenguaje, tecnológica y estética (Román-García; Almansa-Martínez; Cruz- } \\
\text { Díaz, 2016) } \\
\text { Autores y audiencias, mensajes y significados, representación y realidad, } \\
\text { alfabetización mediática (Vraga et al., 2016) }\end{array}$ \\
\hline \multicolumn{3}{|c|}{$\begin{array}{l}\text { Respuestas posibles conforme a escala de Likert: } \\
\text { Totalmente en desacuerdo (1), En desacuerdo (2), Ni de acuerdo ni en desacuerdo (3), De acuerdo (4), Totalmente de acuerdo (5) }\end{array}$} \\
\hline \multicolumn{3}{|c|}{ Variables de clasificación sociodemográficas } \\
\hline \multicolumn{3}{|c|}{$\begin{array}{l}\text { Género } \\
\text { Edad } \\
\text { Área geográfica de residencia habitual } \\
\text { Nivel de estudios reglados finalizados más alto } \\
\text { Área de conocimiento de sus estudios online principales } \\
\text { Situación profesional actual } \\
\text { Número de titulaciones/diplomas de educación online finalizados: nivel de grado y postgrado }\end{array}$} \\
\hline
\end{tabular}


Otra aproximación diferente con el e-learning ha sido llevada a cabo en el área de la alfabetización financiera (Sánchez-Rebull; Campa-Planas; Hernández-Lara, 2011).

Teniendo en cuenta todo lo anterior, el artículo propone una escala para la medición del empoderamiento mediático basado en las competencias de ciudadanía activa y de uso de diversos medios de comunicación, y adquiridas mediante educación online. Para ello se consideran las siguientes hipótesis de trabajo, una principal $(\mathrm{H} 1)$ y dos complementarias ( $\mathrm{H} 2$ y H3), de acuerdo al conjunto de items propuestos para su medición:

- H1: La escala posee validez psicométrica, conforme a su base teórica, estructura factorial de los items propuestos y subescalas: ciudadanía activa y uso diversificado de medios de comunicación.

- H2: La edad adquiere gran importancia en la medición del empoderamiento mediático desarrollado mediante $e$ learning.

- H3: A mayor número de titulaciones obtenidas mediante educación online, mayor empoderamiento mediático obtenido a través de e-learning.

\section{Material y métodos}

\subsection{Diseño de items}

Se diseñó un cuestionario para encuesta online que permitiese poner de manifiesto la adquisición de competencias relacionadas con la ciudadanía activa y el uso de diversos medios de comunicación. En la tabla 1 se pueden observar los ocho items definidos para medir el empoderamiento mediático adquirido mediante educación online. También se incorporan las dimensiones consideradas por varios autores, y que han servido como guías para las definiciones. Finalmente se aprecian otras variables de clasificación tenidas en cuenta durante la investigación.

Los items tienen relación con las dimensiones propuestas:

- la participación ciudadana, la actuación social, la integración comunitaria, la adquisición de conocimientos relativos a la ciudadanía y la creación de contenidos mediáticos, se vinculan con la dimensión de ciudadanía activa. Hay que destacar que este último ítem ayuda a contrastar la orientación mediática de la ciudadanía activa, situación acorde con el uso actual que de los medios sociales vienen realizando los ciudadanos;

- los items que tienen que ver con el uso de más medios de comunicación, conocimiento sobre los mismos, y su utilización eficaz, están enlazados con la dimensión de uso diversificado de los medios de comunicación.

El cuestionario fue contrastado a nivel externo con una profesora de la Universitat de Barcelona experta en metodología de investigación educativa. Se definió que los sujetos de la población de análisis fuesen antiguos alumnos de la Universitat Oberta de Catalunya (UOC). El cuestionario, en formato online mediante la aplicación Google Forms, fue sometido a un proceso de pilotaje durante el mes de febrero de 2016 con alumnos procedentes de la base de datos del Laboratori d'Experimentació del eLearn Center de la UOC.
Tabla 2. Ficha técnica y características sociodemográficas de los resultados. Encuesta online

\begin{tabular}{|c|c|c|}
\hline \multicolumn{3}{|c|}{$\begin{array}{l}\text { Ficha técnica y características sociodemográficas de los resulta- } \\
\text { dos. Encuesta online }\end{array}$} \\
\hline Universo & \multicolumn{2}{|c|}{$\begin{array}{l}\text { Base de datos con } 55.298 \text { antiguos alum- } \\
\text { nos de la UOC }\end{array}$} \\
\hline Selección de la muestra & \multicolumn{2}{|c|}{$\begin{array}{l}\text { Muestreo aleatorio no probabilístico: } \\
\text { invitación mediante mensajes de correo } \\
\text { electrónico }\end{array}$} \\
\hline $\begin{array}{l}\text { Método de recolección } \\
\text { de información }\end{array}$ & \multicolumn{2}{|c|}{$\begin{array}{l}\text { Cuestionario online con preguntas } \\
\text { cerradas }\end{array}$} \\
\hline $\begin{array}{l}\text { Dimensión de la } \\
\text { muestra }\end{array}$ & \multicolumn{2}{|l|}{544 respuestas válidas } \\
\hline Error muestral & \multicolumn{2}{|c|}{$\begin{array}{l}\text { Margen de error del } \pm 4,20 \% \text { para los da- } \\
\text { tos globales, con una población infinita, } \\
\text { en el caso de máxima indeterminación } \\
\text { ( } p=q=50 \%) \text {, y con un nivel de confianza } \\
\text { del } 95,5 \%\end{array}$} \\
\hline $\begin{array}{l}\text { Período de trabajo de } \\
\text { campo }\end{array}$ & \multicolumn{2}{|c|}{5 de mayo - 28 de junio de 2016} \\
\hline Cuotas & \multicolumn{2}{|l|}{ Sin establecer } \\
\hline Ponderación & \multicolumn{2}{|l|}{ No aplicable } \\
\hline Respuesta por género & \multicolumn{2}{|l|}{$\begin{array}{l}\text { Mujeres: } 49,4 \% \\
\text { Hombres: } 50,6 \%\end{array}$} \\
\hline $\begin{array}{l}\text { Edad media del encues- } \\
\text { tado }\end{array}$ & \multicolumn{2}{|l|}{46 años } \\
\hline $\begin{array}{l}\text { Área geográfica de } \\
\text { residencia habitual }\end{array}$ & \multicolumn{2}{|l|}{$\begin{array}{l}\text { Cataluña: } 67,0 \% \\
\text { Resto de España: } 29,0 \% \\
\text { Fuera de España: } 4,0 \%\end{array}$} \\
\hline $\begin{array}{l}\text { Nivel de estudios regla- } \\
\text { dos finalizados más alto }\end{array}$ & \multicolumn{2}{|l|}{$\begin{array}{l}\text { Grado: } 50,9 \% \\
\text { Postgrado: } 49,1 \%\end{array}$} \\
\hline $\begin{array}{l}\text { Área de conocimiento } \\
\text { de sus estudios online } \\
\text { principales }\end{array}$ & $\begin{array}{l}\text { Artes y humanidades } \\
\text { Derecho y ciencias políticas } \\
\text { Economía y empresa } \\
\text { Idiomas } \\
\text { Información y comunicación } \\
\text { Informática, multimedia y } \\
\text { telecomunicaciones } \\
\text { Psicología y educación } \\
\text { Salud } \\
\text { Doctorado } \\
\text { Otros }\end{array}$ & $\begin{array}{r}\% \\
6,4 \\
11,9 \\
27,8 \\
0,4 \\
11,9 \\
\\
18,9 \\
17,1 \\
0,6 \\
2,2 \\
2,8\end{array}$ \\
\hline \multirow{2}{*}{$\begin{array}{l}\text { Situación profesional } \\
\text { actual }\end{array}$} & $\begin{array}{l}\text { No trabaja: } \\
\text {-Desempleado/a } \\
\text {-Inactivo/a } \\
\text {-Estudiante } \\
\text {-Tareas en el hogar } \\
\text {-Otros }\end{array}$ & $\begin{array}{c}\% \\
6,8 \\
0,9 \\
0,5 \\
0,2 \\
2,6\end{array}$ \\
\hline & $\begin{array}{l}\text { Sí trabaja: } \\
\text {-Trabajador/a } \\
\text {-Autónomo/a } \\
\text {-Empresario/a }\end{array}$ & $\begin{array}{r}\% \\
76,7 \\
9,9 \\
2,4\end{array}$ \\
\hline $\begin{array}{l}\text { Número de titulacio- } \\
\text { nes/diplomas de educa- } \\
\text { ción online finalizados }\end{array}$ & \multicolumn{2}{|c|}{$\begin{array}{l}\text { Nivel Grado: Media=1,09 }(D E=0,76) \text { [Míni- } \\
\text { mo, Máximo] }=[0,6] \\
\text { Nivel Postgrado: Media=0,69 }(D E=0,91) \\
{[\text { Mínimo, Máximo] }=[0,6]}\end{array}$} \\
\hline
\end{tabular}




\subsection{Muestra}

Tras el pilotaje y la revisión de los resultados, se contactó con el área UOC Alumni para que pudiesen enviar los urls de los cuestionarios online a los antiguos alumnos UOC y pudiese comenzar el trabajo de campo, que se llevó a cabo durante los meses de mayo y junio de 2016. Se utilizó un cuestionario final redactado en catalán y castellano, para considerar las diferentes preferencias idiomáticas de los alumnos. En la tabla 2 se puede observar la ficha técnica de la encuesta realizada a 55.298 antiguos alumnos de la UOC. La muestra ascendió a 561 respuestas (tasa de respuesta=1,01\%), de las cuales 544 fueron válidas (tasa de respuesta $=0,98 \%$ ). Esta tasa de respuesta - similar a algunas investigaciones actuales llevadas a cabo mediante cuestionarios online a grupos de antiguos alumnos (Head, 2016; Wotherspoon; McCarthy, 2016) - fue compartida y validada por UOC Alumni, que indicó que era la cifra de participación de graduados normalmente obtenida..

\subsection{Análisis de datos}

Fue llevado a cabo con los siguientes programas informáticos: IBM SPSS Statistics for Windows, Version 22.0 (IBM, 2013), $R$ version 3.3.1 ( $R$ Core Team, 2016), y Lavaan 0.5-22 (Rosseel, 2012). Estos programas permitieron la realización de un análisis descriptivo, bivariante, factorial exploratorio, factorial confirmatorio, y de la varianza. Considerando que se encontraron claramente dos factores, no hizo falta tener en cuenta las indicaciones planteadas por Matsunaga (2010) con respecto a utilizar otro tipo de programas informáticos o técnicas estadísticas para seleccionar el número adecuado y más ajustado de factores para la creación de sus correspondientes constructos. Considerando la dimensión de la muestra total $(n=544)$, se impuso su división en dos subconjuntos aleatorios (Rhodes; Arceo, 2004) sobre los que realizar los análisis factorial exploratorio $(n=300)$ y factorial confirmatorio ( $n=244)$.

Dado el número de items planteados (8 items), estas submuestras cumplían diversos requerimientos sobre el número mínimo de individuos a analizar: 150 para análisis factorial exploratorio (Guadagnoli; Velicer, 1988) y factorial confirmatorio (Muthén; Muthén, 2002). Se impusieron dos restricciones más: que la submuestra de población fuese igual o superior a 300 personas, y que la submuestra para el modelo teórico fuese igual o superior a 200 sujetos (Myers; Ahn; Jin, 2011; Rouquette; Falissard, 2011). La tabla 3 muestra los estadísticos descriptivos más comunes obtenidos para los items inspeccionados.

\section{El empoderamiento mediático adquirido mediante educación online puede ser medido mediante items relacionados con la ciudadanía activa y el uso diver- sificado de los medios de comunicación}

\section{Resultados}

\subsection{Escala de empoderamiento mediático mediante e-learning}

Con el objeto de testear la estructura de la escala de empoderamiento mediático (8 items), se llevó a cabo un análisis de componentes principales con rotación Varimax con el primer subconjunto de la muestra extraído aleatoriamente $(n=300)$. El test de Kaiser-Meyer-Olkin $(K M O=0,90)$ y el test de esfericidad de Bartlett $(p=0,000)$ fueron aplicados para comprobar la validez de la muestra, con respecto a la generación de factores basados en los datos. Tras ello se seleccionaron dos factores: ciudadanía activa y uso diversificado de los medios de comunicación, con valores propios superiores a 1, y que permitieron alcanzar un $83,21 \%$ de la varianza total explicada. Los componentes rotados para los dos factores finales fueron superiores a 0,68 , lo que indicaba su significación. En cuanto a sus comunalidades, todas eran superiores a 0,65. La confiabilidad de cada escala fue comprobada mediante la estimación de la consistencia interna que proporciona el alfa de Cronbach, siendo en ambos casos válidas ( $\alpha=0,93$ y $\alpha=0,95$, respectivamente, y $\alpha=0,94$ para el total de los 8 items). La tabla 4 resume los resultados para los dos factores estimados: ciudadanía activa y uso de diversos medios de comunicación.

Al objeto de verificar si los dos constructos basados en estos factores se ajustaban correctamente, se realizó un análisis factorial confirmatorio con el segundo subconjunto obtenido de la muestra $(n=244)$. La estructura factorial de las dos subescalas fue testeada. El modelo obtenido obtuvo unos

Tabla 3. Items de ciudadanía activa y uso diversificado de los medios: media, desviación estándar (DE), skewness y kurtosis ( $\mathrm{n}=544)$.

\begin{tabular}{|c|c|c|c|c|c|}
\hline Dimensión & Items & Media & DE & Skewness & Kurtosis \\
\hline \multirow{5}{*}{ Ciudadanía activa } & Participar más activamente en la vida civil y política & 2,72 & 1,32 & 0,19 & $-1,09$ \\
\hline & $\begin{array}{l}\text { Actuar socialmente frente a aspectos de la vida que veo que no funcio- } \\
\text { nan correctamente }\end{array}$ & 2,83 & 1,32 & 0,09 & $-1,10$ \\
\hline & Sentirme más integrado en grupos, comunidades, asociaciones, etc. & 2,84 & 1,30 & 0,09 & $-1,10$ \\
\hline & Ganar más conocimiento sobre mis derechos como ciudadano & 3,00 & 1,35 & $-0,08$ & $-1,18$ \\
\hline & Crear mensajes y contenidos propios, relacionados con las noticias & 3,00 & 1,32 & $-0,08$ & $-1,12$ \\
\hline \multirow{3}{*}{$\begin{array}{l}\text { Uso diversificado de } \\
\text { medios de comunicación }\end{array}$} & Usar más medios de comunicación & 3,26 & 1,26 & $-0,27$ & $-0,91$ \\
\hline & Conocer más medios de comunicación & 3,19 & 1,29 & $-0,21$ & $-1,02$ \\
\hline & Utilizar eficazmente los medios de comunicación & 3,26 & 1,24 & $-0,30$ & $-0,81$ \\
\hline
\end{tabular}


Tabla 4. Análisis factorial exploratorio (matriz de componentes rotados) ( $n=300$ ) y análisis factorial confirmatorio (matriz de estimaciones) ( $n=244)$. Subescalas de ciudadanía activa y uso diversificado de los medios: media, desviación estándar, skewness y kurtosis ( $\mathrm{n}=544$ ).

\begin{tabular}{|c|c|c|c|c|c|c|}
\hline \multirow[t]{2}{*}{ Dimensión } & \multicolumn{2}{|r|}{ Items } & \multicolumn{2}{|c|}{$\begin{array}{l}\text { Análisis factorial } \\
\text { exploratorio }\end{array}$} & \multicolumn{2}{|c|}{$\begin{array}{l}\text { Análisis factorial } \\
\text { confirmatorio }\end{array}$} \\
\hline & & & F1 & $\mathbf{F} 2$ & F1 & F2 \\
\hline \multirow{5}{*}{ Ciudadanía activa } & \multicolumn{2}{|c|}{ Participar más activamente en la vida civil y política } & 0,89 & & $1,00 * * *$ & \\
\hline & \multicolumn{2}{|c|}{$\begin{array}{l}\text { Actuar socialmente frente a aspectos de la vida que veo que no } \\
\text { funcionan correctamente }\end{array}$} & 0,88 & & $0,95^{* * *}$ & \\
\hline & \multicolumn{2}{|c|}{ Sentirme más integrado en grupos, comunidades, asociaciones, etc. } & 0,85 & & $0,91^{* * *}$ & \\
\hline & \multicolumn{2}{|c|}{ Ganar más conocimiento sobre mis derechos como ciudadano } & 0,79 & & $0,97^{* * *}$ & \\
\hline & \multicolumn{2}{|c|}{ Crear mensajes y contenidos propios, relacionados con las noticias } & 0,69 & & $0,77^{* * *}$ & \\
\hline \multirow{3}{*}{$\begin{array}{l}\text { Uso diversificado de } \\
\text { medios de } \\
\text { comunicación }\end{array}$} & \multicolumn{2}{|c|}{ Usar más medios de comunicación } & & 0,91 & & $1,00^{* * *}$ \\
\hline & \multicolumn{2}{|c|}{ Conocer más medios de comunicación } & & 0,90 & & $0,99 * * *$ \\
\hline & \multicolumn{2}{|c|}{ Utilizar eficazmente los medios de comunicación } & & 0,88 & & $0,96 * * *$ \\
\hline \multicolumn{7}{|c|}{ Estadísticos } \\
\hline \multicolumn{3}{|c|}{$\begin{array}{l}\text { KMO (Kaiser-Meyer-Olkin) } \\
\text { Prueba } X^{2} \text { de esfericidad de Bartlett } \\
\text { Grados de libertad } \\
\text { p-valor } \\
\text { Comunalidades }\end{array}$} & \multicolumn{2}{|c|}{$\begin{array}{c}0,90 \\
2.331,87 \\
28 \\
0,000 \\
>0,65\end{array}$} & & \\
\hline \multicolumn{3}{|l|}{$\begin{array}{l}\text { Varianza }(\%) \\
\text { Del total }\end{array}$} & \multicolumn{2}{|c|}{$\begin{array}{c}69,2313,98 \\
83,21\end{array}$} & & \\
\hline \multicolumn{3}{|c|}{$\begin{array}{l}\text { Coeficientes de fiabilidad (alfa de Cronbach): De factores } \\
\text { Del total }\end{array}$} & \multicolumn{2}{|c|}{$\begin{array}{l}0,930,95 \\
0,94\end{array}$} & & \\
\hline & & & \multicolumn{2}{|c|}{$\begin{array}{c}\mathrm{CFI}=0,98 ; \mathrm{TLI}=0,98 ; \\
\mathrm{RMSEA}=0,09 ; \\
\mathrm{SRMR}=0,04 ; \\
\mathrm{NNFI}=0,98 ; \mathrm{RFI}=0,96 ; \\
\mathrm{NFI}=0,98 ; \mathrm{IFI}=0,98\end{array}$} \\
\hline \multicolumn{2}{|l|}{ Escalas } & Media & \multicolumn{2}{|c|}{ DE } & Skewness & Kurtosis \\
\hline \multicolumn{2}{|c|}{$\begin{array}{l}\text { F1: Ciudadanía activa } \\
\text { F2: Uso diversificado de medios de comuni- } \\
\text { cación }\end{array}$} & $\begin{array}{l}2,88 \\
3,24\end{array}$ & \multicolumn{2}{|c|}{$\begin{array}{l}1,17 \\
1,21\end{array}$} & $\begin{array}{c}0,04 \\
-0,27\end{array}$ & $\begin{array}{l}-0,96 \\
-0,83\end{array}$ \\
\hline
\end{tabular}

estadísticos que ajustaban el modelo teórico de un modo adecuado $\left(\chi^{2}=53,48\right.$, $p$-valor $=0,000 ; \mathrm{CFI}=0,98 ; \mathrm{TLI}=0,98$; RM$\mathrm{SEA}=0,09)$. En la tabla 4 pueden verse también todos estos aspectos de un modo sintético, así como el hecho de que la subescala de uso diversificado de medios de comunicación obtiene una puntuación mayor que la subescala de ciudadanía activa (media de 3,24 frente a 2,88, respectivamente). La figura 1 muestra el modelo estructural de la escala de empoderamiento mediática validada a través del uso de estas dos subescalas. Con todo ello se confirmó la hipótesis 1.

Existe una vinculación y un territorio común por explorar en materia de educación online, educomunicación, empoderamiento, y ciudadanía activa

\subsection{Análisis descriptivo y bivariante de las subescalas}

Tras la definición y validación de las subescalas, se llevó a cabo su análisis descriptivo conforme a las variables sociodemográficas diseñadas. A nivel general todos los valores medios de los grupos fueron superiores para la subescala de uso diversificado de medios de comunicación, frente a la subescala de ciudadanía activa. Resultó interesante comprobar que las personas que no trabajaban señalaban poseer un mayor valor medio en ambas subescalas. Mediante un análisis de la varianza también se observó que las diferencias más notables, y las únicas significativas, se daban entre los grupos formados por la variable edad: las personas de mayor edad alcanzaban unas puntuaciones superiores en ambas subescalas. Esto confirmó la hipótesis 2. La tabla 5 expone estos resultados.

A la vista de estos datos se realizó un análisis bivariante que permitió detectar las correlaciones estadísticamente significativas entre las variables (se tuvo en cuenta la correlación Rho de Spearman $\left(r_{s}\right)$ para variables ordinales y parejas de variables continuas y ordinales). De este modo se comprobó que ambas subescalas -ciudadanía activa y uso diversificado de los medios- estaban relacionadas significativamente de un modo positivo $(r=0,68, p=0,000)$, y que un mayor número de titulaciones online de grado proporcionaban un incremento significativo en ambas subescalas ( $r=0,12$ y $r=0,11, p<0,01$, respectivamente), mientras que un número mayor de titulaciones online de postgrado contribuía significativamente solamente en la subescala de uso diversificado de los medios de comunicación $(r=0,10, p<0,05)$. Estos aspectos permitieron confirmar la hipótesis 3 . 


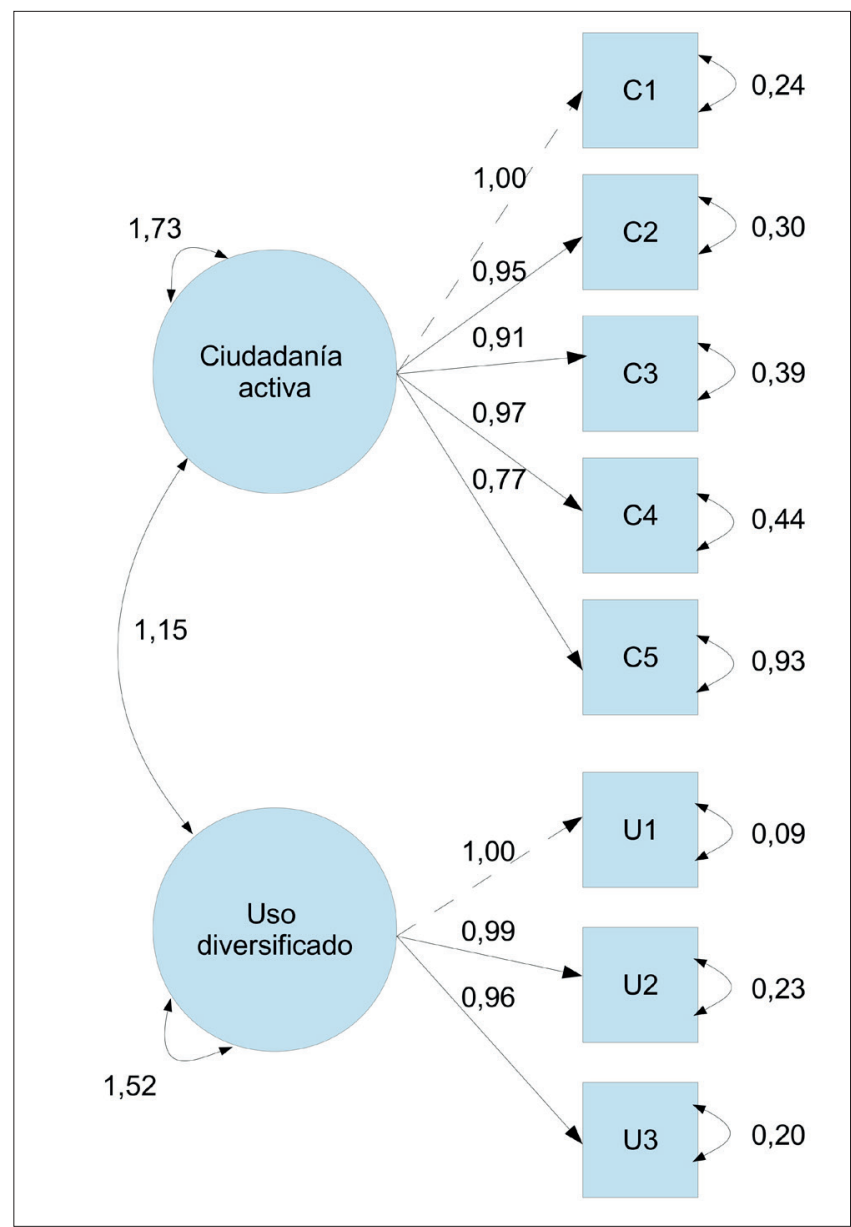

Figura 1. Modelo estructural de la escala de empoderamiento mediático basada en dos subescalas: ciudadanía activa y uso diversificado de los medios de comunicación

Se observó que la variable de edad era la que más influía sobre el resto, contribuyendo también a confirmar la hipótesis 2 , y relacionándose positivamente con ambas subescalas, sobre todo con la de uso diversificado de los medios $\left(r_{s}=0,12\right.$ y $r_{s}=0,21, p<0,01$ y $p=0,000$, respectivamente), así como con el número de títulos online de grado y postgrado $\left(r_{s}=0,09\right.$, $p<0,05$, para ambas subescalas), y las personas de género masculino $(r=0,14, p<0,01)$. Se comprobó que los hombres solían tener un número mayor de postgrados online $(r=0,12$, $p<0,01)$. También se detectó que las personas con un mayor nivel de estudios tenían trabajo $\left(r_{s}=0,15, p<0,01\right)$, y que claramente preferían seguir postgrados online frente a cursar estudios de grado online $\left(r_{s}=0,67\right.$ y $r_{s}=-0,17$, respectivamente, $p=0,000$ en ambos casos). Estos resultados aparecen en la tabla 6.

Existe una escala con validez psicométrica para la medición del empoderamiento mediático obtenido mediante e-learning, basado en una subescala de ciudadanía activa y otra de uso diversificado de los medios

\section{Discusión y conclusiones}

En primer lugar hay que hacer constar ciertas limitaciones del trabajo. La realización mediante cuestionario online autocumplimentado de una investigación cuantitativa relacionada con el comportamiento de los propios sujetos de estudio, no siempre se ajusta a la realidad (Wright, 2005). Además la muestra de antiguos estudiantes de la UOC no puede ser generalizada a todos los tipos de educación online, tanto formales como no formales. Respecto a la confiabilidad y validez del instrumento, se hacen necesarias más comprobaciones con otros estudiantes como, por ejemplo, con los que han realizado estudios mediante educación presencial, que actuarían como grupo de control. Así, futuras investigaciones podrían tanto cuestionar otras dimensiones como estimar la importancia relativa de las variables sociodemográficas aquí analizadas, en cuanto a la estimación de las subescalas planteadas. Otro aspecto tiene relación con el hecho de que una de las hipótesis complementarias de la investigación sea correlacional (Hernández-Sampieri; Fernández-Collado; BaptistaLucio, 2014), lo que supone que no se pueda establecer una relación causal entre las respectivas variables, aspecto que queda pendiente para próximas investigaciones.

Tabla 5. Escalas de ciudadanía activa y uso diversificado de los medios y características sociodemográficas de los antiguos estudiantes online (valores medios de las escalas) $(n=544)$

\begin{tabular}{|c|c|c|c|}
\hline \multicolumn{2}{|c|}{ Variables } & \multirow{2}{*}{$\begin{array}{c}\text { Ciudadanía activa } \\
2,80 \\
2,95\end{array}$} & \multirow{2}{*}{$\begin{array}{c}\text { Uso diversificado } \\
3,16 \\
3,32\end{array}$} \\
\hline Género & $\begin{array}{l}\text { Mujer } \\
\text { Hombre }\end{array}$ & & \\
\hline Grupo de edad & $\begin{array}{l}\text { Inferior a } 35 \text { años } \\
35-44 \text { años } \\
45-54 \text { años } \\
\text { Superior a } 54 \text { años }\end{array}$ & $\begin{array}{l}2,88 \\
2,73(a) \\
2,85(b) \\
3,33(a)(b)\end{array}$ & $\begin{array}{l}2,92(c) \\
2,99(d)(e) \\
3,34(d)(f) \\
3,75(c)(e)(f)\end{array}$ \\
\hline Nivel de estudios reglados finalizados más alto & $\begin{array}{l}\text { Grado/Licenciatura/Diplomatura } \\
\text { Postgrado (Masters, etc.) }\end{array}$ & $\begin{array}{l}2,91 \\
2,84\end{array}$ & $\begin{array}{l}3,33 \\
3,14\end{array}$ \\
\hline Situación profesional actual & $\begin{array}{l}\text { No trabaja } \\
\text { Trabaja }\end{array}$ & $\begin{array}{l}3,12 \\
2,85\end{array}$ & $\begin{array}{l}3,43 \\
3,22\end{array}$ \\
\hline \multicolumn{4}{|c|}{$\begin{array}{l}\text { Considerando varianzas iguales (test de Levene), existen diferencias significativas entre las medias de los grupos que poseen las siguientes codifi } \\
\text { caciones: } \\
\text { (d),(f) }=p<0,05 \\
\text { (a),(b),(c) }=p<0,01 \\
\text { (e) }=p=0,000\end{array}$} \\
\hline
\end{tabular}


Tabla 6. Medias, desviaciones estándar y correlaciones entre las escalas y diversas variables observadas $(n=544)$

\begin{tabular}{|c|c|c|c|c|c|c|c|c|c|c|}
\hline & $M$ & DE & (1) & (2) & (3) & (4) & (5) & (6) & (7) & (8) \\
\hline Ciudadanía activa (1) & 2,88 & 1,17 & - & & & & & & & \\
\hline Uso diversificado (2) & 3,24 & 1,21 & $0,68^{* * *}$ & - & & & & & & \\
\hline $\begin{array}{l}\text { Número de titulaciones/diplomas } \\
\text { de educación online finalizados: } \\
\text { nivel de grado (3) }\end{array}$ & 1,09 & 0,76 & $0,12^{* *}$ & $0,11^{* *}$ & - & & & & & \\
\hline $\begin{array}{l}\text { Número de titulaciones/diplomas } \\
\text { de educación online finalizados: } \\
\text { nivel de postgrado (4) }\end{array}$ & 0,69 & 0,91 & 0,07 & $0,10^{*}$ & 0,00 & - & & & & \\
\hline Género (5) & 0,51 & 0,50 & 0,07 & 0,07 & 0,01 & $0,12^{* *}$ & - & & & \\
\hline Grupo de edad (6) & 1,64 & 0,81 & $0,12^{* *}$ & $0,21^{* * *}$ & $0,09^{*}$ & $0,09^{*}$ & $0,14^{* *}$ & - & & \\
\hline $\begin{array}{l}\text { Nivel de estudios reglados finali- } \\
\text { zados más alto (7) }\end{array}$ & 0,49 & 0,50 & $-0,02$ & $-0,07$ & $-0,17^{* * *}$ & $0,67^{* * *}$ & 0,00 & $-0,11^{*}$ & - & \\
\hline Situación profesional actual (8) & 0,89 & 0,31 & $-0,07$ & $-0,06$ & $-0,05$ & 0,05 & 0,04 & $-0,02$ & $0,15^{* *}$ & - \\
\hline \multicolumn{11}{|l|}{${ }^{*} p<0,05 ;{ }^{* *} p<0,01 ;{ }^{* * *} p=0,000$} \\
\hline \multicolumn{11}{|c|}{$\begin{array}{l}\text { Uso diversificado }(1)=\{1=\text { Totalmente en desacuerdo, } 2=\text { En desacuerdo, } 3=\text { Ni de acuerdo ni en desacuerdo, } 4=\text { De acuerdo, } 5=\text { Totalmente de acuerdo }\} \\
\text { Ciudadanía activa }(2)=\{1=\text { Totalmente en desacuerdo, } 2=\text { En desacuerdo, } 3=\text { Ni de acuerdo ni en desacuerdo, } 4=\text { De acuerdo, } 5=\text { Totalmente de acuerdo }\} \\
\text { Género }(5)=\{0=\text { Mujer, } 1=\text { Hombre }\} \\
\text { Grupo de edad }(6)=\{0=\text { Inferior a } 35,1=35-44 \text { años, } 2=45-54 \text { años, } 3=\text { Superior a } 54\} \\
\text { Nivel de estudios reglados finalizados más alto }(7)=\{0=\text { Grado/Licenciatura/Diplomatura, } 1=\text { Postgrado (Masters, etc.) }\} \\
\text { Situación profesional actual }(8)=\{0=\text { No trabaja, } 1=\text { Sí trabaja }\}\end{array}$} \\
\hline
\end{tabular}

Pese a ello, el trabajo realiza una destacada contribución académica al enriquecer la investigación sobre empoderamiento digital. Concretamente, mediante una aproximación basada en la adquisición mediante educación online de competencias relacionadas con la ciudadanía activa y el uso diversificado de medios de comunicación. La investigación realiza una conceptualización teórica de aspectos sobre empoderamiento y educación mediática, la ciudadanía activa, y el e-learning. Sintetizando investigaciones anteriores destinadas a la validación psicométrica de escalas, se propone un instrumento de medición que es confirmado y que puede ser utilizado para futuras investigaciones. Además se exponen los resultados de la aplicación de las subescalas conforme a diversas variables sociodemográficas, y que confirman la gran importancia de disponer de una mayor edad en materia de empoderamiento ciudadano.

La educación online permite adquirir competencias para ejercer una ciudadanía activa y puede contribuir al empoderamiento de las personas en riesgo de exclusión social

Los resultados de esta investigación proporcionan pruebas empíricas sobre el empoderamiento mediático adquirido por los estudiantes de e-learning. Se ha comprobado que a mayor número de certificaciones obtenidas mediante cursos online, mayor desarrollo de competencias y habilidades válidas para la mejora del empoderamiento mediático y ciudadano de las personas. Su carácter, orientado hacia la ciudadanía activa y el uso diversificado de los medios, puede contribuir a mejorar la situación de determinados sectores de la población con riesgo de exclusión social. En una situación en la que los propios estudiantes de periodismo tienen unas competencias mediáticas limitadas (Schmidt, 2015), la formación a distancia a través de internet puede contribuir a mejorar determinadas habilidades y destrezas útiles para el conjunto de los ciudadanos, en relación con la adquisición de una mayor capacidad de ejercicio individual de los valores democráticos.

Bajo esta perspectiva, el e-learning basado en los medios sociales puede contribuir al crecimiento de una educación horizontal y dialógica (Freire, 1970), potenciando las capacidades de interconexión de sus alumnos, y contribuyendo a su empoderamiento (Hergueta-Covacho; Marta-Lazo; Gabelas-Barroso, 2016). Se consolidaría así una manera de enseñar que piensa en fortalecer personalmente a los estudiantes mediante el aprovechamiento de los medios:

“Una educación -sea ella presencial o a través de medios- capaz de responder a los desafíos formativos contemporáneos, habrá de proponerse activar las potencialidades de autoaprendizaje y coaprendizaje que se encuentran latentes en sus destinatarios y estimular la gestión autónoma de los educandos en su 'aprender-aaprender', en su propio camino hacia el conocimiento: la observación personal, la confrontación y el intercambio, el cotejo de alternativas, el razonamiento crítico, la elaboración creativa" (Kaplún, 2002, p. 206).

En última instancia el empoderamiento mediático planteado, con sus dimensiones de ciudadanía y uso diversificado de los medios, permitiría obtener a lo largo de la vida y mediante los instrumentos asociados a la educación online, una ciudadanía que no se desvinculase de contrastar y usar eficientemente las noticias que recibe de un modo cada día más expansivo, pero en "espacios discursivos cada vez más reducidos" (Gozálvez-Pérez, 2011, p. 131). Sin duda alguna los beneficios de todo ello son cada día más necesarios. 


\section{Agradecimientos}

Los autores agradecen a Julià Minguillón, Francesc Santanach y a su equipo en el Laboratori d'Experimentació del eLearn Center de la UOC, concretamente a Antoni Bertran, su colaboración y soporte durante el diseño de la investigación. Del mismo modo reconocen el apoyo de Carles Rocadembosch y su equipo en la UOC Alumni, especialmente el de Laura Tello, en el trabajo de campo. Finalmente, agradecen la colaboración externa de la profesora Ruth Vilà Baños, de la Universitat de Barcelona, durante el proceso de diseño del cuestionario

\section{Bibliografía}

Akkoyunlu, Buket; Yilmaz-Soylu, Meryem; Caglar, Mehmet (2010). "A study on developing 'digital empowerment scale' for university students". HU Journal of education, v. 39, pp. 10-19. https://goo.gl/5QoYQU

Arke, Edward; Primack, Brian (2009). "Quantifying media literacy: development, reliability, and validity of a new measure". Educational media international, v. 46, n. 1, pp. 53-65. https://goo.gl/KnmPRI

https://doi.org/10.1080/09523980902780958

Ashley, Seth; Maksl, Adam; Craft, Stephanie (2013). "Developing a news media literacy scale". Journalism \& mass communication educator, v. 68, n. 1, pp. 7-21.

http://scholarworks.boisestate.edu/cgi/viewcontent.cgi?art icle $=1053 \&$ context $=$ communication facpubs

https://doi.org/10.1177/1077695812469802

Becker, Gary S. (1993). Human capital: a theoretical and empirical analysis, with special reference to education. New York: National Bureau of Economic Research: Distributed by Columbia University Press. ISBN: 0226041093

http://papers.nber.org/books/beck75-1

Bier, Melinda; Schmidt, Spring; Shields, David; Zwarun, Lara; Sherblom, Stephen; Primack, Brian; Pulley, Cynthia; Rucker, Billy (2013). "School-based smoking prevention with media literacy: A pilot study". Journal of media literacy education, v. 2, n. 3, pp. 185-198.

http://digitalcommons.uri.edu/jmle/vol2/iss3/1

Celot, Paolo; Pérez-Tornero, José-Manuel (2009). Study on assessment criteria for media literacy levels. Brussels: European Association for Viewers' Interests (EAVI).

http://ec.europa.eu/culture/library/studies/literacy-criteriareport_en.pdf

Chang, Chiung-Sui; Liu, Eric-Zhi-Feng; Lee, Chun-Yi; Chen, Nian-Shing; Hu, Da-Chian; Lin, Chun-Hung (2011). "Developing and validating a media literacy self-evaluation scale (MLSS) for elementary school students". Turkish online journal of educational technology (Tojet), v. 10, n. 2, pp. 63-71. http://eric.ed.gov/?id=EJ932226

Choi, Moonsun (2016). "A concept analysis of digital citizenship for democratic citizenship education in the internet age". Theory \& research in social education, v. 44, n. 4. https://doi.org/10.1080/00933104.2016.1210549

Claro, Magdalena; Preiss, David D.; San-Martín, Ernesto; Jara, Ignacio; Hinostroza, J.-Enrique; Valenzuela, Susana;
Cortés, Flavio; Nussbaum, Miguel (2012). “Assessment of $21^{\text {st }}$ century ICT skills in Chile: Test design and results from high school level students". Computers \& education, v. 59, n. 3, pp. 1042-1053.

https://goo.gl/lbAOwV

https://doi.org/10.1016/j.compedu.2012.04.004

Dias-Fonseca, Tânia; Potter, John (2016). "Media education as a strategy for online civic participation in Portuguese schools". Comunicar, v. 24, n. 49, pp. 09-17. https://doi.org/10.3916/C49-2016-01

Duran, Robert L.; Yousman, Bill; Walsh, Kaitlin M.; Longshore, Melanie A. (2008). "Holistic media education: An assessment of the effectiveness of a college course in media literacy". Communication quarterly, v. 56, n. 1, pp. 49-68. https://doi.org/10.1080/01463370701839198

European Union (2016). EUR-Lex http://eur-lex.europa.eu/homepage.html

Eurostat (2016). "People at risk of poverty or social exclusion by age and sex". Eurostat. Your key to European statistics. http://ec.europa.eu/eurostat/en/web/products-datasets/-/ ILC_PEPSO1

Ferrés-Prats, Joan; Piscitelli, Alejandro (2012). "Media competence. Articulated proposal of dimensions and indicators". Comunicar, v. 19, n. 38, pp. 75-82. https://doi.org/10.3916/c38-2012-02-08

Freire, Paulo (1970). Pedagogía del oprimido. México, D.F.: Siglo Veintiuno Editores. ISBN: 9788432301841

http://www.servicioskoinonia.org/biblioteca/general/ FreirePedagogiadelOprimido.pdf

Gil-Quintana, Javier (2016). "La nueva realidad smooc: una ventana abierta a la educomunicación / The new social smooc reality, an open window to media literacy". Revista mediterránea de comunicación / Mediterranean journal of communication, v. 7, n. 2, pp. 59-68.

https://doi.org/10.14198/medcom2016.7.2.5

Gozálvez-Pérez, Vicent; Aguaded-Gómez, Ignacio (2012). "Educación para la autonomía en sociedades mediáticas". Anàlisi: quaderns de comunicació i cultura, v. 45, pp. 1-14. https://ddd.uab.cat/pub/analisi/analisi_a2012m6n45/ analisi a2012m6n45p1.pdf https://doi.org/10.7238/a.v0i45.1326

Gozálvez-Pérez, Vicent (2011). “Eduación para la ciudadanía democrática en la cultura digital". Comunicar, v. 18, n. 36, pp. 131-138.

https://doi.org/10.3916/C36-2011-03-04

Guadagnoli, Edward; Velicer, Wayne-F. (1988). "Relation of sample size to the stability of component patterns". Psychological bulletin, v. 103, n. 2, pp. 265-275.

https://doi.org/10.1037/0033-2909.103.2.265

Head, Alison J. (2016). Staying Smart: How Today's Graduates Continue to Learn Once They Complete College. Rochester, NY: Social Science Research Network, SSRN Scholarly Paper: 2712329 https://doi.org/10.2139/ssrn.2573090

Hergueta-Covacho, Elisa; Marta-Lazo, Carmen; Gabelas- 
Barroso, José-Antonio (2016). “Educación mediática e inteRmetodología relacional aplicada a los mooc / Media literacy and relational inter-methodology implementation on mooc". Revista mediterránea de comunicación / Mediterranean journal of communication, v. 7, n. 2, pp. 47-58. https://doi.org/10.14198/medcom2016.7.2.4

Hernández-Sampieri, Roberto; Fernández-Collado, Carlos; Baptista-Lucio, Pilar (2014). Metodología de la investigación (6th ed.). México, D.F.: McGraw-Hill Education. ISBN: 9781456223960

Hobbs, Renee (2011). "Empowering learners with digital and media literacy". Knowledge quest, v. 39, n. 5, pp. 12-17. https://goo.gl/VVBGQg

Hur, Mann-Hyung; Im, Yeonwook (2013). "The influence of e-learning on individual and collective empowerment in the public sector: An empirical study of Korean government employees". The international review of research in open and distributed learning, v. 14, n. 4, pp. 191-213.

http://www.irrodl.org/index.php/irrodl/article/view/1498

IBM Corp. (2013). IBM SPSS Statistics for Windows, Version 22.0. Armonk, NY: IBM Corp.

Kaplún, Mario (2002). Una pedagogía de la comunicación (el comunicador popular). La Habana: Editorial Caminos. ISBN: 9597070359

http://perio.un/p.edu.ar/catedras/system/files/kaplun-el_ comunicador_popular_0.pdf

Koc, Mustafa; Barut, Esra (2016). “Development and validation of new media literacy scale (NMLS) for university students". Computers in human behavior, v. 63, pp. 834-843. https://doi.org/10.1016/j.chb.2016.06.035

Kratochvil, Jiří (2014). "Efficiency of e-learning in an information literacy course for medical students at the Masaryk University". The electronic library, v. 32, n. 3, pp. 322-340. https://doi.org/10.1108/EL-07-2012-0087

Lenger, Alexander; Schumacher, Florian (2015). "The global configurations of inequality: Stratification, glocal inequalities, and the global social structure". En: Lenger, Alexander; Schumacher, Florian (eds.). Understanding the dynamics of global inequality. Social exclusion, power shift, and structural changes. Springer, pp. 3-46. ISBN: 9783662447666 https://goo.gl/KvVhRP https://doi.org/10.1007/978-3-662-44766-6_1

Levin-Zamir, Diane; Lemish, Dafna; Gofin, Rosa (2011). "Media health literacy (MHL): Development and measurement of the concept among adolescents". Health education research, v. 26, n. 2, pp. 323-335.

https://goo.gl/EGCMrN

https://doi.org/10.1093/her/cyr007

Mäkinen, Maarit (2006). "Digital empowerment as a process for enhancing citizens' participation". E-learning and digital media, v. 3, n. 3, pp. 381-395.

http://ldm.sagepub.com/content/3/3/381.full.pdf+html https://doi.org/10.2304/elea.2006.3.3.381

Maksl, Adam; Ashley, Seth; Craft, Stephanie (2015). “Measuring news media literacy". Journal of media literacy edu- cation, v. 6, n. 3, pp. 29-45. http://digitalcommons.uri.edu/jmle/vol6/iss3/3

Martens, Hans (2013). "Evaluating media literacy education: Concepts, theories and future directions". Journal of media literacy education, v. 2, n. 1, pp. 1-22. http://digitalcommons.uri.edu/jmle/vol2/iss1/1

Martínez-Pérez, Jorge (2016). "El empoderamiento del alumnado desde el modelo sMOOC / Student's empowerment from the sMOOC model". Revista mediterránea de comunicación / Mediterranean journal of communication, v. 7, n. 2, pp. 69-79.

https://doi.org/10.14198/medcom2016.7.2.6

Matsunaga, Masaki (2010). "How to factor-analyze your data right: do's, don'ts, and how-to's". International journal of psychological research, v. 3, n. 1, pp. 97-110.

http://www.redalyc.org/articulo.oa?id=299023509007

Montero, Maritza (2003). Teoría y práctica de la psicología comunitaria. La tensión entre comunidad y sociedad. Buenos Aires: Paidós. ISBN: 9501245187

https://goo.gl/ogJR2Q

Moreno-Pulido, Alexis; Sánchez-Fernández, Rosa (2015). "Papel de la biblioteca en la enseñanza de competencias informacionales en posgrados de la Universidad Nacional de Educación a Distancia (UNED)". El profesional de la información, v. 24, n. 5, pp. 665-673.

https://doi.org/10.3145/epi.2015.sep.15

Muthén, Linda K.; Muthén, Bengt O. (2002). “How to use a Monte Carlo study to decide on sample size and determine power". Structural equation modeling: A multidisciplinary journal, v. 9, v. 4, pp. 599-620.

https://goo.gl/nGZjtL

https://doi.org/10.1207/S15328007SEM0904_8

Myers, Nicholas D.; Ahn, Soyeon; Jin, Ying (2011). “Sample size and power estimates for a confirmatory factor analytic model in exercise and sport: A Monte Carlo approach". Research quarterly for eExercise and sport, v. 82, n. 3, pp. 412-423.

https://www.statmodel.com/download/Myers, $\% 20$ Ahn,\%20\&\%20Jin,\%202011.pdf

https://doi.org/10.1080/02701367.2011.10599773

$\mathrm{Ng}$, Wan (2012). "Can we teach digital natives digital literacy?". Computers \& education, v. 59, n. 3, pp. 1065-1078. https://doi.org/10.1016/j.compedu.2012.04.016

Norman, Cameron D.; Skinner, Harvey A. (2006). "eHEALS: The eHealth literacy scale". Journal of medical internet research, v. 8, n. 4, pp. E27.

https://doi.org/10.2196/jmir.8.4.e27

Onah, Bernardine-Ifeona (2012). Development and validation of an electronic learning (e-learning) programme for the digital empowerment of students in South-East, Nigeria. Thesis. University of Nigeria, Nsukka.

Park, Sora (2012). "Dimensions of digital media literacy and the relationship with social exclusion". Media international Australia, v. 142, pp. 87-100.

https://goo.gl/BJOCiu

https://doi.org/10.1177/1329878X1214200111 
Pérez-Rodríguez, María-Amor; Delgado-Ponce, Águeda (2012). "From digital and audiovisual competence to media competence: Dimensions and indicators". Comunicar, v. 20, n. 39, pp. 25-34.

https://doi.org/10.3916/C39-2012-02-02

Phelps-Tschang, Jane; Miller, Elizabeth; Rice, Kristen; Primack, Brian (2015). "Web-based media literacy to prevent tobacco use among high school students". Journal of media literacy education, v. 7, n. 3, pp. 29-40.

http://digitalcommons.uri.edu/jmle/vol7/iss $3 / 3$

Piaget, Jean (2000). “Commentary on Vygotsky's criticisms of language and thought of the child and judgement and reasoning in the child". New ideas in psychology, v. 18, n. 2-3, pp. 241-259. https://goo.gl/XFXQRO

https://doi.org/10.1016/S0732-118X(00)00012-X

Pineda-Báez, Clelia; Hennig-Manzouli, Cristina; SegoviaCifuentes, Yasbley; Díaz-Gómez, Darwin; Sánchez-Duarte, Mónica; Otero, María-Patricia; Rees, Geraint-Paul (2012). "Alfabetización informacional en la educación superior virtual: logros y desafíos". Informational literacy in virtual higher education: Achievements and challenges, n. 26, pp. 83-104. https://dialnet.unirioja.es/servlet/articulo?codigo $=3985357$

Potter, James (2010). "The state of media literacy". Journal of broadcasting \& electronic media, v. 54, n. 4, pp. 675-696. https://doi.org/10.1080/08838151.2011.521462

Prior, Daniel D.; Mazanov, Jason; Meacheam, David; Heaslip, Graham; Hanson, James (2016). "Attitude, digital literacy and self efficacy: Flow-on effects for online learning behavior". The Internet and higher education, v. 29, pp. 91-97. https://goo.gl/qviCRB

https://doi.org/10.1016/j.iheduc.2016.01.001

$R$ Core Team (2016). $R$ : A language and environment for statistical computing. Viena, Austria: R Foundation for Statistical Computing.

http://www.R-project.org

Redecker, Christine; Leis, Miriam; Leendertse, Matthijs; Punie, Yves; Gijsbers, Govert; Kirschner, Paul; Stoyanov, Slavi; Hoogveld, Bert (2011). The future of learning: Preparing for change. Luxembourg: Publications Office of the European Union. ISBN: 9789279214721

http://ftp.jrc.es/EURdoc/JRC66836.pdf

Rhodes, Scott D.; Arceo, Ramiro (2004). "Developing and testing measures predictive of hepatitis $A$ vaccination in a sample of men who have sex with men". Health education research, v. 19, n. 3, pp. 272-283.

https://doi.org/10.1093/her/cyg026

Rivoltella, Pier-Cesare (2005). Media education: fondamenti didattici e prospettive di ricerca. Brescia: La Scuola. ISBN: 9788835018247

Román-García, Sara; Almansa-Martínez, Ana; Cruz-Díaz, María-del-Rocío (2016). "Adultos y mayores frente a las TIC. La competencia mediática de los inmigrantes digitales". Comunicar, v. 24, n. 49, pp. 101-110. https://doi.org/10.3916/C49-2016-10

Rosseel, Yves (2012). “lavaan: An R package for structural equa- tion modeling". Journal of statistical software, v. 48, n. 2, 36 pp. https://doi.org/10.18637/jss.v048.i02

Rouquette, Alexandra; Falissard, Bruno (2011). "Sample size requirements for the internal validation of psychiatric scales". International journal of methods in psychiatric research, v. 20, n. 4, pp. 235-249.

https://goo.gl/f2ppRN

https://doi.org/10.1002/mpr.352

Sánchez-García, Sandra; Yubero-Jiménez, Santiago (2015). "Función social de las bibliotecas públicas: nuevos espacios de aprendizaje y de inserción social". El profesional de la información, v. 24, n. 2, pp. 103-112.

https://doi.org/10.3145/epi.2015.mar.03

Sánchez-Rebull, María-Victoria; Campa-Planas, Fernando; Hernández-Lara, Ana B. (2011). "Dolceta, educación online para los consumidores: módulo de alfabetización financiera en España". El profesional de la información, v. 20, n. 6, pp. 682-689. https://doi.org/10.3145/epi.2011.nov.13

Schmidt, Hans C. (2015). "More than writing and reporting: Examining the overall media literacy of today's journalism students". Teaching journalism \& mass communication, v. 5, n. 1, pp. 43-56.

https://goo.gl/dCUXRE

Schultz, Theodore W. (1961). "Investment in human capital". The American economic review, v. 51, n. 1, pp. 1-17.

Tait, Alan (2013). "Distance and e-learning, social justice, and development: The relevance of capability approaches to the mission of open universities". International review of research in open and distance learning, v. 14, n. 4.

http://www.irrodl.org/index.php/irrodl/article/view/1526

Tang, Yingqi; Tseng, Hung-Wei (2013). “Distance learners' self-efficacy and information literacy skills". The journal of academic librarianship, v. 39, n. 6, pp. 517-521.

https://goo.gl/EenYgN

https://doi.org/10.1016/j.acalib.2013.08.008

Tury, Sandra; Robinson, Lyn; Bawden, David (2015). “The information seeking behaviour of distance learners: A case study of the University of London international programmes". The journal of academic librarianship, v. 41, n. 3, pp. 312-321. http://openaccess.city.ac.uk/11937

https://doi.org/10.1016/j.acalib.2015.03.008

Unesco (1982). "Grunwald declaration on media education". En: International symposium on media education. Grunwald, Germany.

http://www.unesco.org/education/information/standards/ english/world_unesco.htm

Van-de-Vord, Rebecca (2010). “Distance students and online research: Promoting information literacy through media literacy". The internet and higher education, v. 13, n. 3, pp. 170-175. https://goo.gl/XAdXqm https://doi.org/10.1016/j.iheduc.2010.03.001

Varis, Tapio; Puukko, Mika (eds.) (2010). Challenges of global elearning. Tampere: Tampere University, Research Centre for Vocational Education. ISBN: 9789514480379 https://goo.gl/dSycJM 
Vraga, Emily; Tully, Melissa; Kotcher, John; Smithson, Anne-Bennett; Broeckelman-Post, Melissa (2016). "A multidimensional approach to measuring news media literacy". Journal of media literacy education, v. 7, n. 3, pp. 41-53. http://digitalcommons.uri.edu/jmle/vol7/iss3/4

Vygotsky, Lev-Semyonovic (1997). Educational psychology. CRC Press. ISBN: 9781878205155

Wotherspoon, Sheena E.; McCarthy, Peter W. (2016). "The factors and motivations behind United Kingdom chiropractic professional association membership: a survey of the Welsh Institute of Chiropractic Alumni". Chiropractic \& manual therapies, v. 24, n. 35, pp. 1-9. https://doi.org/10.1186/s12998-016-0115-x

Wright, Kevin B. (2005). "Researching internet-based populations: Advantages and disadvantages of online survey research, online questionnaire authoring software packages, and web survey services". Journal of computer-mediated communication, v. 10, n. 3.

https://doi.org/10.1111/j.1083-6101.2005.tb00259.x

Zhang, Hui; Zhu, Chang (2016). "A study of digital media literacy of the $5^{\text {th }}$ and $6^{\text {th }}$ grade primary students in Beijing". The Asia-Pacific education researcher, v. 25, n. 4, pp. 579592.

https://doi.org/10.1007/s40299-016-0285-2

\section{EPI cumple 25 años}

\section{2 febrero}

Aparece el $n^{\circ} 1$ de Information world en español (IWE), publicado por la editorial inglesa Learned Information. Se redacta en España pero se imprime y distribuye desde Oxford. ISSN: 0965-3821

\section{7 enero-febrero}

Se publica en Barcelona, pero como revista de la editorial holandesa Swets \& Zeitlinger, que compra la cabecera y la lista de suscriptores a Learned Information. En el contrato se especifica que se permite usar el título sólo durante un año.

Se añade el subtítulo "La revista del profesional de la información".

La numeración cambia a la forma volumen/número: sale el v. 6, n. 1-2.

\section{8 enero-febrero}

Cambia el título a El profesional de la información, pero se mantiene el logotipo IWE. "Information world en español" consta en la cabecera como pequeño subtítulo.

Además de continuar con noticias y reportajes, comienza a publicar artículos revisados por un comité científico y poco a poco la revista va siendo indizada por diversas bases de datos nacionales y extranjeras. Nuevo ISSN: 1386-6710. Sale el v. 7, n. 1-2.

\section{9 septiembre}

El título sólo es El profesional de la información. En otros lugares de la revista se usan ocasionalmente las siglas EPI.

2000 enero-febrero

La revista empieza a distribuirse online en formato pdf.

\section{2 enero}

EPl adopta la periodicidad bimestral (6 números al año).

\section{4 enero}

EPI pasa a ser una revista de la editorial Taylor \& Francis como resultado de la compra de Swets \& Zeitlinger Publishers por parte de dicha editorial el 6 de noviembre de 2003. Metapress (grupo Ebsco) distribuye la versión electrónica.

\section{5 enero}

Después de 13 años de rodaje en manos de editoriales inglesas y holandesa, el equipo directivo de $E P /$ ve claro que los suscriptores van a recibir mejor servicio si todos los procesos de edición y de distribución se centralizan en España; por lo que, previo acuerdo con la editorial Taylor \& Francis, se constituye en editorial, con sede en Barcelona.

2006 enero

EPI comienza a ser indizada por la base de datos Social Science Citation Index (Web of Science), de Thomson ISI y por Scopus, de Elsevier.

\section{7 noviembre}

EPI comienza a utilizar el programa Open Journal System (OJS) del Repositorio Español de Ciencia y Tecnología (Recyt) para gestionar los manuscritos. Recyt es un proyecto de la Fundación Española para la Ciencia y la Tecnología (Fecyt). EPI es una de las 3 primeras revistas seleccionadas por Fecyt para poner en marcha Recyt.

2007 diciembre

EPI organiza el primer Spanish Meeting Point en la feria de la Online Information Conference lantes Online Information Meeting). Se trata de un stand que ayuda a empresas y académicos españoles a participar en el entorno internacional de la industria de la información.

\section{0 junio}

Se publican los Journal Citation Reports (JCR) de 2009 de Thomson Reuters, en los que aparece por primera vez EPI con un Factor de Impacto (IF) de 0,475.

\section{1 mayo}

EPI-ThinkEPI organiza en Barcelona la $1^{\text {a }}$ jornada sobre Calidad de las revistas de ciencias sociales (CRECS), que se celebrará anualmente desde entonces en ciudades distintas.

\section{1 noviembre}

Inicio de la colección de libros El profesional de la información, en colaboración con la Editorial UOC.

\section{5 enero}

EPI deja de publicarse en papel. Los suscriptores acceden a los contenidos desde el Recyt de la Fecyt.

\section{6 enero}

Se convoca el I Premio Scimago-EPI al mejor artículo de investigación publicado en la revista en los últimos 6 años.
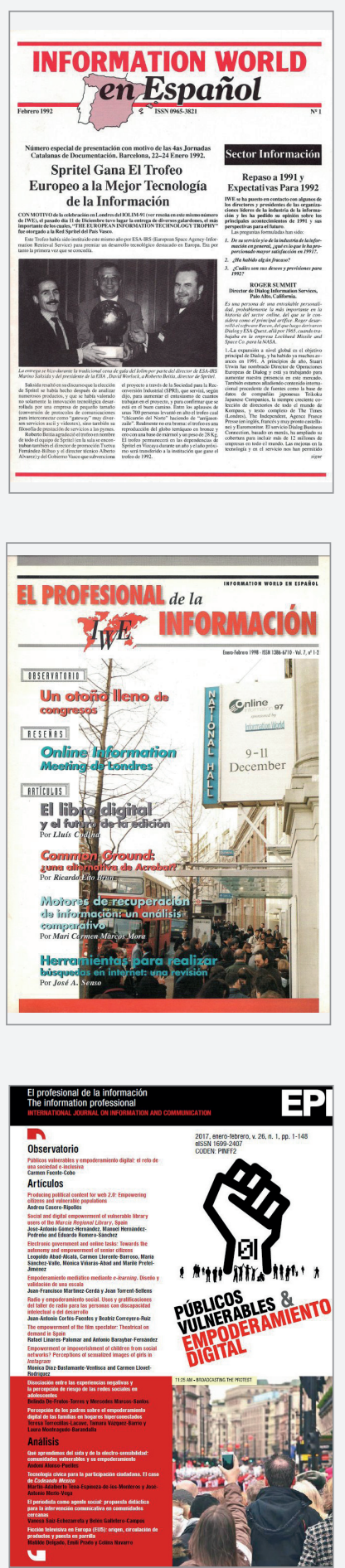